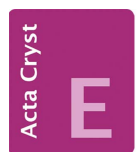

CRYSTALLOGRAPHIC COMMUNICATIONS

ISSN 2056-9890

Received 7 March 2016

Accepted 24 March 2016

Edited by V. V. Chernyshev, Moscow State University, Russia

Keywords: crystal structure; 3D Hirshfeld analysis; 1,3-dimethylbarbituric acid; anticonvulsant activity; hypnotic activity.

CCDC reference: 1444879

Supporting information: this article has supporting information at journals.iucr.org/e

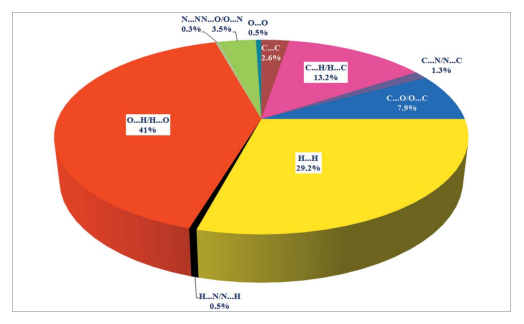

OPEN $\odot$ ACCESS

\section{Isoquinolinium 5-(2,4-dinitrophenyl)-1,3-dimethyl- 2,6-dioxo-1,2,3,6-tetrahydropyrimidin-4-olate: crystal structure, Hirshfeld surface analysis and pharmacological evaluation}

\author{
Ponnusamy Poornima Devi and Doraisamyraja Kalaivani*
}

PG and Research Department of Chemistry, Seethalakshmi Ramaswami College, Tiruchirappalli 620 002, Tamil Nadu, India. *Correspondence e-mail: kalaivbalaj@yahoo.co.in

The asymmetric unit of the title salt $\mathrm{C}_{9} \mathrm{H}_{8} \mathrm{~N}^{+} \cdot \mathrm{C}_{12} \mathrm{H}_{9} \mathrm{~N}_{4} \mathrm{O}_{7}^{-}$, which exhibits anticonvulsant and hypnotic activities, comprises one anion and one cation interacting via an $\mathrm{N}-\mathrm{H} \cdots \mathrm{O}$ hydrogen bond. In the anion, the six-membered rings are inclined each to other at $42.78(9)^{\circ}$. The nitro groups in the 2,4dinitrophenyl fragment attached to the aromatic ring in the para and ortho positions are twisted from its plane by 3.1 (2) and $45.5(2)^{\circ}$, respectively. In the crystal, weak $\mathrm{C}-\mathrm{H} \cdots \mathrm{O}$ hydrogen bonds consolidate the crystal packing. The Hirshfeld surface analysis revealed that $\mathrm{O} \cdots \mathrm{H} / \mathrm{H} \cdots \mathrm{O}$ intermolecular contacts predominate in the crystal packing.

\section{Chemical context}

Barbiturates play a significant role in biological systems (Hueso-Ureña et al., 2003). Epilepsy (convulsion) is a lifethreatening neurological disorder which requires immediate treatment with suitable drugs (Shorvon, 2004). Barbiturates have been proved to be potent drugs for this dreadful disorder (Nadkarni et al., 2005). The isoquinoline unit also displays a wide spectrum of activity and it is an important component of many biologically active alkaloids (Montalban, 2011). Since 2008, we have been periodically synthesizing new barbiturate derivatives and exploring their anticonvulsant activity (Kalaivani et al., 2008; Kalaivani \& Malarvizhi, 2009; Kalaivani \& Buvaneswari, 2010; Manickkam \& Kalaivani, 2011; Babykala \& Kalaivani, 2012; Buvaneswari \& Kalaivani, 2013; Vaduganathan \& Doraisamyraja, 2014; Gomathi \& Kalaivani, 2015). The title molecular salt, which is a new derivative of 1,3dimethylbarbituric acid (barbiturate), was recently obtained by our group. Herewith we report its crystal structure.<smiles>Cn1c(O)c(-c2c([O-])ccc3ccccc23)c(=O)n(C)c1=O</smiles> 


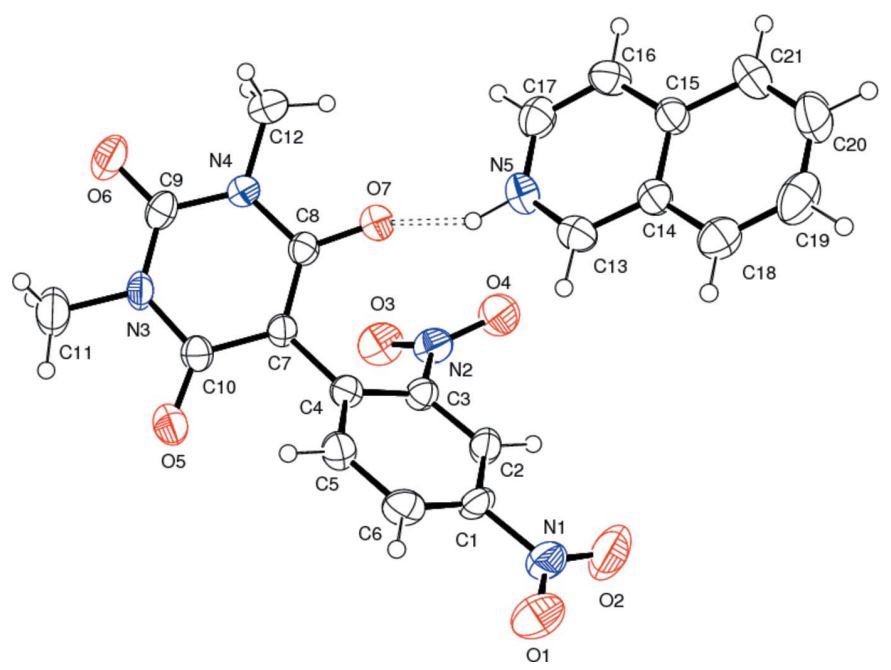

Figure 1

The asymmetric unit of (I) showing the atom numbering and $40 \%$ probability displacement ellipsoids. The doubled-dashed line denotes the $\mathrm{N}-\mathrm{H} \cdots \mathrm{O}$ hydrogen bond between the cation and anion.

\section{Structural commentary}

In the title compound, (I) (Fig. 1), all the bond lengths and bond angles are normal and comparable with those observed in the related barbiturates (Sridevi \& Kalaivani, 2012; Gunaseelan \& Doraisamyraja, 2014). The plane of the dinitroaromatic ring $\mathrm{C} 1-\mathrm{C} 6$ and that of the barbiturate ring $\mathrm{C} 7 / \mathrm{C} 8 /$ $\mathrm{N} 4 / \mathrm{C} 9 / \mathrm{N} 3 / \mathrm{C} 10$ form a dihedral angle of $42.78(9)^{\circ}$. The nitro groups in the 2,4-dinitrophenyl fragment attached to the aromatic ring in the para and ortho positions are twisted from its plane by 3.1 (2) and $45.5(2)^{\circ}$, respectively. Thus the para nitro group is more involved in delocalizing the negative charge than the ortho nitro group in the anionic part. This sort of delocalization of the charge over a large area imparts a maroon red colour to the title compound.

\section{Supramolecular features}

The aminium group is involved in formation of an $\mathrm{N}-\mathrm{H} \cdots \mathrm{O}$ hydrogen bond (Table 1) between the isoquinolinium cation $(\mathrm{N} 5-\mathrm{H} 5 A)$ and the deprotonated enol oxygen atom O7. In the crystal, weak $\mathrm{C}-\mathrm{H} \cdots \mathrm{O}$ hydrogen bonds (Table 1) consolidate the crystal packing (Fig. 2). An $R_{2}^{1}(6)$ motif is generated by the $\mathrm{C}-\mathrm{H}$ groups [C13- $\mathrm{H} 13$ and $\mathrm{C} 20-\mathrm{H} 20]$ of the isoquinolinium cation and oxygen atom $\mathrm{O} 5$ of the carbonyl group of the barbiturate ring of the anion. Although there are three rings with cyclically delocalized $\pi$ electron clouds, no $\pi-\pi$ stacking interactions are observed between them.

\section{3D Hirshfeld Surface Analysis and 2D Fingerprint Analysis}

Hirshfeld surfaces (Spackman \& Jayatilaka, 2009) and the associated 2D-fingerprint plots (McKinnon et al., 2007) of the title molecular salt have been generated using Crystal Explorer 3.1 (Wolff et al., 2013). Hirshfeld surfaces mapped
Table 1

Hydrogen-bond geometry $\left(\AA{ }^{\circ}\right)$.

\begin{tabular}{lllll}
\hline$D-\mathrm{H} \cdots A$ & $D-\mathrm{H}$ & $\mathrm{H} \cdots A$ & $D \cdots A$ & $D-\mathrm{H} \cdots A$ \\
\hline $\mathrm{N} 5-\mathrm{H} 5 A \cdots \mathrm{O} 7$ & $0.93(6)$ & $1.74(6)$ & $2.592(6)$ & $150(5)$ \\
$\mathrm{C} 13-\mathrm{H} 13 \cdots \mathrm{O} 5^{\mathrm{i}}$ & 0.93 & 2.40 & $3.260(7)$ & 153 \\
$\mathrm{C} 16-\mathrm{H} 16 \cdots 6^{\mathrm{ii}}$ & 0.93 & 2.33 & $3.187(7)$ & 154 \\
$\mathrm{C} 17-\mathrm{H} 17 \cdots \mathrm{O} 2^{\mathrm{iii}}$ & 0.93 & 2.61 & $3.424(8)$ & 146 \\
\hline Symmetry codes: & (i) & $x+\frac{1}{2},-y+\frac{1}{2},-z+1 ;$ & (ii) & $-x+3, y-\frac{1}{2},-z+\frac{1}{2} ;$
\end{tabular}

$-x+\frac{5}{2},-y, z-\frac{1}{2}$.

with different properties, e.g. $d_{\mathrm{e}}, d_{\mathrm{norm}}, d_{\mathrm{i}}$, shapeindex, curvedness, have proven to be a useful visualization tool for the analysis of intermolecular interactions. The $2 \mathrm{D}$-fingerprint plots of Hirshfeld surfaces have been used to pinpoint and scrutinize the percentage of hydrogen-bonding interactions present in the crystal structure. The presented graphical plots use the same red-white-blue color scheme, wherein red highlights the shortest intermolecular atomic contacts (negative $d_{\text {norm }}$ values), white is used for contacts around the van der Waals separation, and blue corresponds to longer ones (positive $d_{\text {norm }}$ values). Hirshfeld surface analysis of the new barbiturate of present interest has $d_{\text {norm }}$ values ranging from -0.723 (red) to 1.464 (blue), as specified in Fig. 3. The globularity value (a measure of the degree to which the surface area differs from that of the shape) is less than 1

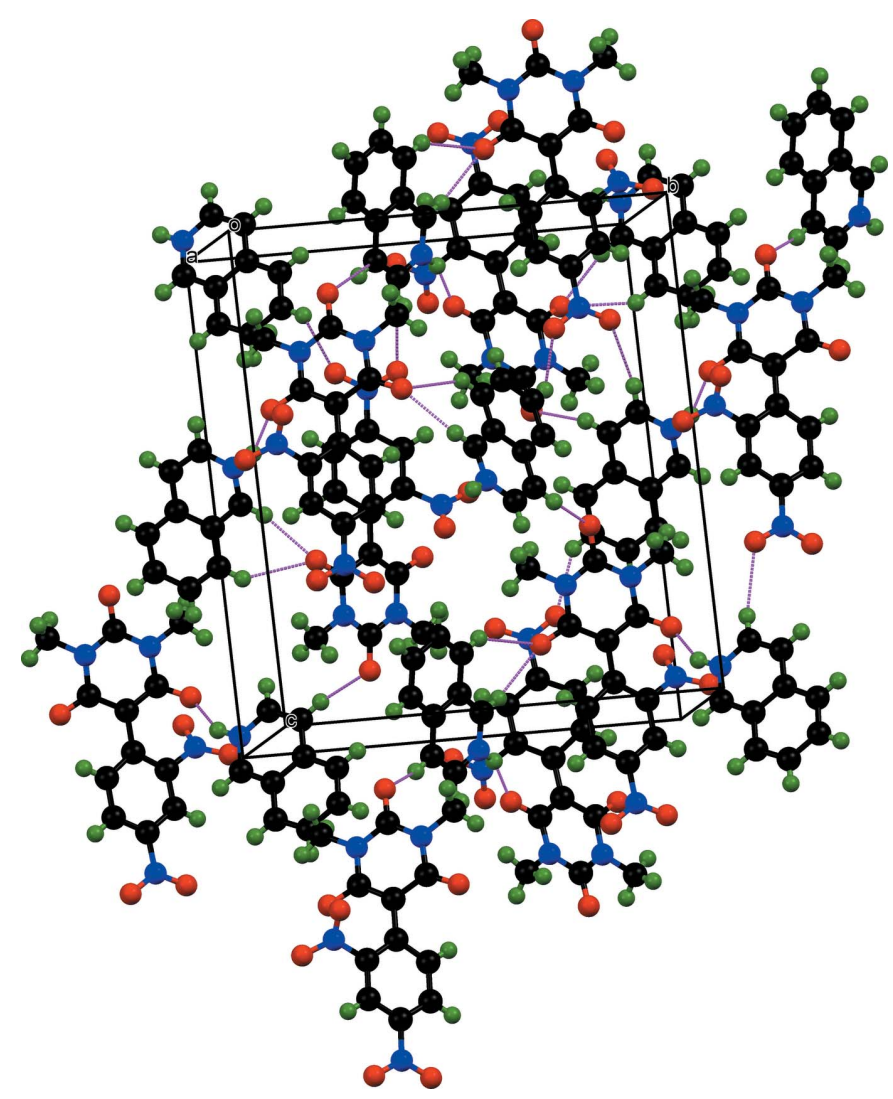

Figure 2

Crystal packing of (I) viewed approximately down the $a$ axis. Hydrogen bonds are shown as purple dotted lines. 


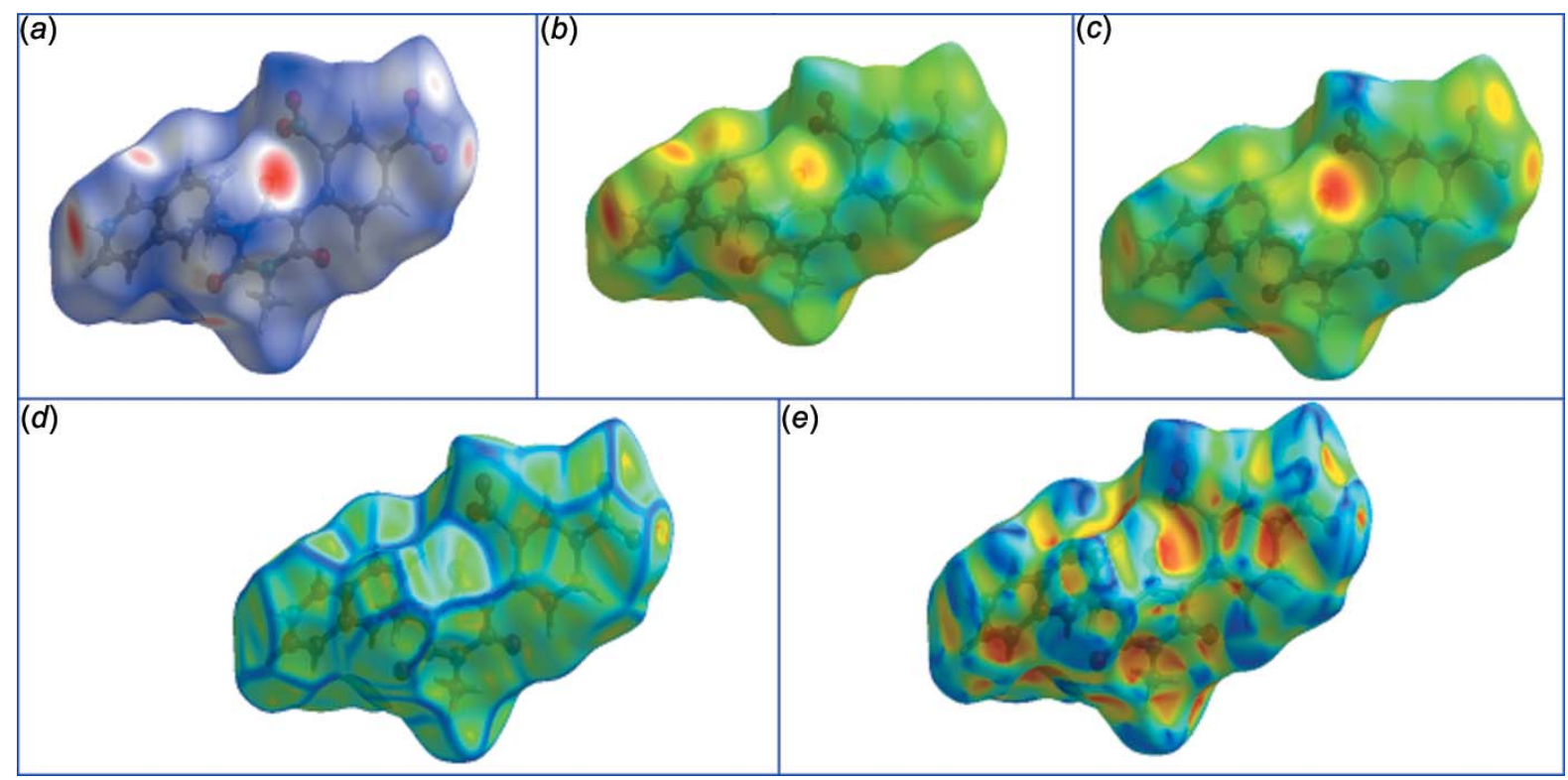

Figure 3

3D Hirshfeld surface analysis of (I) mapped over $(a) d_{\text {norm }}$ ranging from -0.723 (red) to 1.464 (blue); $(b) d_{\mathrm{e}} ;(c) d_{\mathrm{i}} ;(d)$ curvedness; $(e)$ shapeindex.

(0.743), implying a more structured molecular surface and it is an oblate object (asphericity, 0.282). 2D-Fingerprint plots showing contributions from different contacts: $(a)$ overall interactions $(b) \mathrm{C} \cdots \mathrm{H} / \mathrm{H} \cdots \mathrm{C}(c) \mathrm{C} \cdots \mathrm{O} / \mathrm{O} \cdots \mathrm{C}(d) \mathrm{H} \cdots \mathrm{H}(e)$ $\mathrm{O} \cdots \mathrm{H} / \mathrm{H} \cdots \mathrm{O}(f) \mathrm{N} \cdots \mathrm{O} / \mathrm{O} \cdots \mathrm{N}$ are depicted in Fig. 4, and Fig. 5 (pie chart) clearly demonstrates that the $\mathrm{O} \cdots \mathrm{H} / \mathrm{H} \cdots \mathrm{O}$ interactions dominate in the crystal.

\section{Pharmacological activity}

Epilepsy affects about $0.5 \%$ of the world's population. A seizure is caused by an asynchronous high-frequency discharge of a group of neurons, starting locally and spreading to a varying extent to affect other parts of the brain. 1,3-Dimethylbarbituric acid is the most significant compound

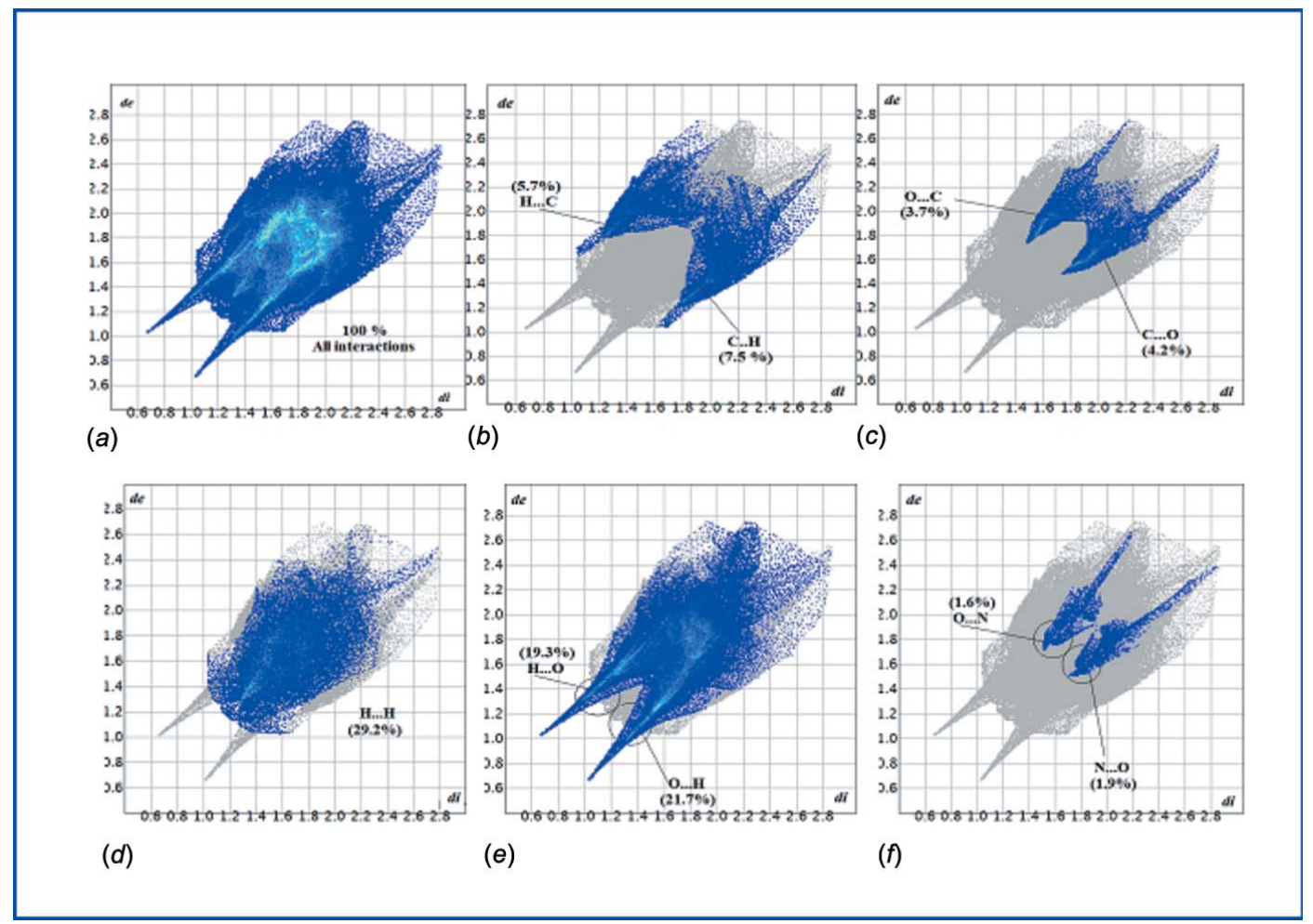

Figure 4

2D Fingerprint plots showing contributions from different contacts: $(a)$ overall interactions; $(b) \mathrm{C} \cdots \mathrm{H} / \mathrm{H} \cdots \mathrm{C} ;(c) \mathrm{C} \cdots \mathrm{O} / \mathrm{O} \cdots \mathrm{C} ;(d) \mathrm{H} \cdots \mathrm{H} ;(e) \mathrm{O} \cdots \mathrm{H} /$ $\mathrm{H} \cdots \mathrm{O}$ and $(f) \mathrm{N} \cdots \mathrm{O} / \mathrm{O} \cdots \mathrm{N}$. 


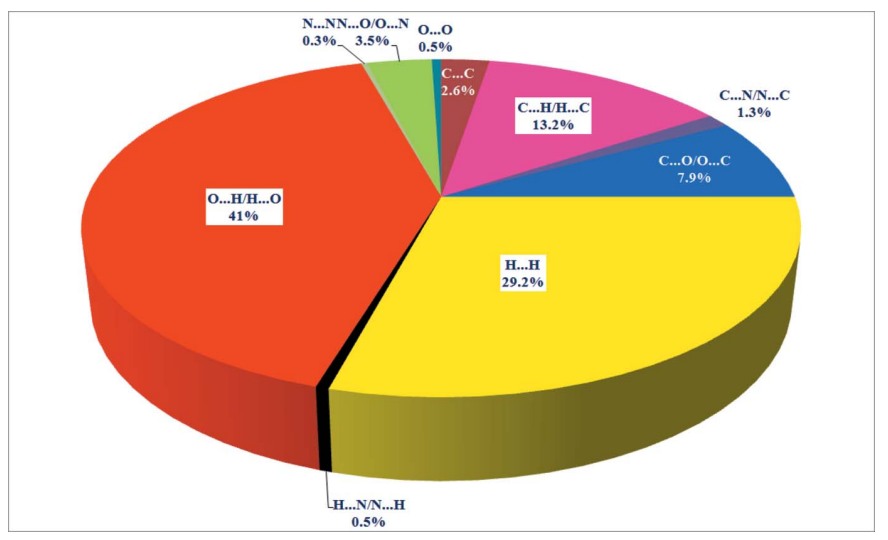

Figure 5

Pie chart showing the quantitative distribution of intermolecular interactions in (I).

with a heterocyclic structure and exists in two tautomeric forms (keto and enol) due to the mobility of active methylene group hydrogen atoms in its molecule. Barbiturates are drugs that act as central nervous system depressants and can therefore produce a wide spectrum of effects from mild sedation to total anaesthesia. They are also effective as anxiolytics, hypnotics and anticonvulsants. As the molecular salt of the present investigation is a derivative of 1,3-dimethylbarbituric acid, it has been subjected to the Maximal Electro Shock method to evaluate its anticonvulsant activity (Misra et al.,1973; Kulkarni, 1999). It reduces all phases of convulsion (tonic-flexor, tonic-extensor, clonic-convulsion and stupor) even at low dosage $\left(25 \mathrm{mg} \mathrm{kg}^{-1}\right)$ and the animals recovered after the experiment.

\section{Synthesis and crystallization}

1-Chloro-2,4-dinitrobenzene $(2.02 \mathrm{~g}, 0.01 \mathrm{~mol})$ in $40 \mathrm{~mL}$ of absolute alcohol was mixed with 1,3-dimethylbarbituric acid $(1.56 \mathrm{~g}, 0.01 \mathrm{~mol})$ in $30 \mathrm{~mL}$ ethanol. To this mixture, $0.02 \mathrm{~mol}$ of isoquinoline was added and the mixture was shaken well for $5 \mathrm{~h}$ and kept as such for $24 \mathrm{~h}$. Excess ethanol was removed through evaporation. A maroon-red pasty mass was obtained. This paste was digested with hot ethanol to obtain a maroonred solid. The solid deposited at the bottom of the flask was filtered, powdered well using an agate mortar, washed again with $20 \mathrm{~mL}$ of dry ether and recrystallized from absolute alcohol. Good quality single crystals suitable for X-ray diffraction analysis were obtained by slow evaporation of ethanol at room temperature (yield: 80\%; m.p. $413 \mathrm{~K}$ ).

\section{Refinement}

Crystal data, data collection and structure refinement details are summarized in Table 2. The $\mathrm{N}$-bound $\mathrm{H}$ atom was located in a difference Fourier map and refined isotropically. C-bound $\mathrm{H}$ atoms were positioned geometrically and refined as riding, with $\mathrm{C}-\mathrm{H}=0.93-0.96 \AA$ and $U_{\text {iso }}(\mathrm{H})=1.2-1.5 U_{\text {eq }}(\mathrm{C})$.
Table 2

Experimental details.

Crystal data

Chemical formula

$M_{\mathrm{r}}$

Crystal system, space group

Temperature (K)

$a, b, c(\AA)$

$V\left(\AA^{3}\right)$

$Z$

Radiation type

$\mu\left(\mathrm{mm}^{-1}\right)$

Crystal size (mm)

Data collection

Diffractometer

Absorption correction

$T_{\min }, T_{\max }$

No. of measured, independent and observed $[I>2 \sigma(I)]$ reflections

$R_{\text {int }}$

$(\sin \theta / \lambda)_{\max }\left(\AA^{-1}\right)$

$\mathrm{C}_{9} \mathrm{H}_{8} \mathrm{~N}^{+} \cdot \mathrm{C}_{12} \mathrm{H}_{9} \mathrm{~N}_{4} \mathrm{O}_{7}^{-}$

451.40

Orthorhombic, $P 22_{1} 2_{1} 2_{1}$

296

7.5315 (3), 15.5640 (8), 17.3901 (8)

2038.47 (16)

4

Mo $K \alpha$

0.11

$0.35 \times 0.30 \times 0.25$

Refinement

$R\left[F^{2}>2 \sigma\left(F^{2}\right)\right], w R\left(F^{2}\right), S$

No. of reflections

No. of parameters

$\mathrm{H}$-atom treatment

$\Delta \rho_{\max }, \Delta \rho_{\min }\left(\mathrm{e} \AA^{-3}\right)$

Absolute structure

Bruker Kappa APEXII CCD

Multi-scan ( $S A D A B S$; Bruker, 2004)

$0.958,0.984$

$27633,3588,2797$

0.038

0.594

Absolute structure parameter

$0.055,0.164,1.11$

3588

302

$\mathrm{H}$ atoms treated by a mixture of independent and constrained refinement

$0.29,-0.26$

Flack $x$ determined using 1033 quotients $\left[\left(I^{+}\right)-\left(I^{-}\right)\right] /\left[\left(I^{+}\right)+\left(I^{-}\right)\right]$ (Parsons et al., 2013)

$0.5(4)$

Computer programs: APEX2, SAINT and XPREP (Bruker, 2004), SIR92 (Altomare $e t$ al., 1993), SHELXL2014 (Sheldrick, 2015), ORTEP-3 for Windows (Farrugia, 2012) and Mercury (Macrae et al., 2008).

\section{Acknowledgements}

The authors are thankful to the DST for financial support, the SAIF, IIT Madras, Chennai - 36, for the single crystal XRD data collection and KMCH College of Pharmacy, Coimbatore, for the anticonvulsant activity results.

\section{References}

Altomare, A., Cascarano, G., Giacovazzo, C. \& Guagliardi, A. (1993). J. Appl. Cryst. 26, 343-350.

Babykala, R. \& Kalaivani, D. (2012). Acta Cryst. E68, o541.

Bruker (2004). APEX2, SAINT, XPREP and SADABS. Bruker AXS Inc., Madison, Wisconsin, USA.

Buvaneswari, M. \& Kalaivani, D. (2013). J. Chem. Crystallogr. 43, 561-567.

Farrugia, L. J. (2012). J. Appl. Cryst. 45, 849-854.

Gomathi, J. \& Kalaivani, D. (2015). Acta Cryst. E71, 723-725.

Gunaseelan, S. \& Doraisamyraja, K. (2014). Acta Cryst. E70, o110201103.

Hueso-Ureña, F., Illán-Cabeza, N. A., Moreno-Carretero, M. N., Martínez-Martos, J. M. \& Ramírez-Expósito, M. J. (2003). J. Inorg. Biochem. 94, 326-334.

Kalaivani, D. \& Buvaneswari, M. (2010). Recent Advances in Clinical Medicine, pp. 255-260. Cambridge, UK: WSEAS Publications.

Kalaivani, D. \& Malarvizhi, R. (2009). Acta Cryst. E65, o2548.

Kalaivani, D., Malarvizhi, R. \& Subbalakshmi, R. (2008). Med. Chem. Res. 17, 369-373. 
Kulkarni, S.K. (1999). Handbook of Experimental Pharamacology, p. 131. Mumbai: Vallabh Prakashan.

Macrae, C. F., Bruno, I. J., Chisholm, J. A., Edgington, P. R., McCabe, P., Pidcock, E., Rodriguez-Monge, L., Taylor, R., van de Streek, J. \& Wood, P. A. (2008). J. Appl. Cryst. 41, 466-470.

Manickkam, V. \& Kalaivani, D. (2011). Acta Cryst. E67, 03475.

McKinnon, J. J., Jayatilaka, D. \& Spackman, M. A. (2007). Chem. Commun. pp. 3814-3816.

Misra, A. K., Dandiya, P. C. \& Kulkarni, S. K. (1973). Indian J. Pharmacol. 5, 449-450.

Montalban, A. G. (2011). Heterocycles in Natural Product Synthesis, pp. 299-339.

Nadkarni, S., LaJoie, J. \& Devinsky, O. (2005). Neurology, 64, S2-11.
Parsons, S., Flack, H. D. \& Wagner, T. (2013). Acta Cryst. B69, 249259.

Sheldrick, G. M. (2015). Acta Cryst. C71, 3-8.

Shorvon, S. D. (2004). The Treatment of Epilepsy. Oxford: Blackwell Publishers.

Spackman, M. A. \& Jayatilaka, D. (2009). CrystEngComm, 11, 1932.

Sridevi, G. \& Kalaivani, D. (2012). Acta Cryst. E68, o1044.

Vaduganathan, M. \& Doraisamyraja, K. (2014). Acta Cryst. E70, 256258.

Wolff, S. K., Grimwood, D. J., McKinnon, J. J., Turner, M. J., Jayatilaka, D. \& Spackman, M. A. (2013). Crystal Explorer. University of Western Australia. 


\section{supporting information}

Acta Cryst. (2016). E72, 570-574 [https://doi.org/10.1107/S2056989016005004]

Isoquinolinium 5-(2,4-dinitrophenyl)-1,3-dimethyl-2,6-dioxo-1,2,3,6-tetrahydro-

pyrimidin-4-olate: crystal structure, Hirshfeld surface analysis and pharmacological evaluation

\section{Ponnusamy Poornima Devi and Doraisamyraja Kalaivani}

Computing details

Data collection: APEX2 (Bruker, 2004); cell refinement: APEX2 and SAINT (Bruker, 2004); data reduction: SAINT and XPREP (Bruker, 2004); program(s) used to solve structure: SIR92 (Altomare et al., 1993); program(s) used to refine structure: SHELXL2014 (Sheldrick, 2015); molecular graphics: ORTEP-3 for Windows (Farrugia, 2012) and Mercury (Macrae et al., 2008); software used to prepare material for publication: SHELXL2014 (Sheldrick, 2015).

Isoquinolinium 5-(2,4-dinitrophenyl)-1,3-dimethyl-2,6-dioxo-1,2,3,6-tetrahydropyrimidin-4-olate

Crystal data

$\mathrm{C}_{9} \mathrm{H}_{8} \mathrm{~N}^{+} \cdot \mathrm{C}_{12} \mathrm{H}_{9} \mathrm{~N}_{4} \mathrm{O}_{7}^{-}$

$M_{r}=451.40$

Orthorhombic, $P 2{ }_{1} 2_{1} 2_{1}$

$a=7.5315(3) \AA$

$b=15.5640(8) \AA$

$c=17.3901(8) \AA$

$V=2038.47(16) \AA^{3}$

$Z=4$

$F(000)=936$

\section{Data collection}

Bruker Kappa APEXII CCD diffractometer

Radiation source: fine-focus sealed tube Graphite monochromator

$\omega$ and $\varphi$ scan

Absorption correction: multi-scan

(SADABS; Bruker, 2004)

$T_{\min }=0.958, T_{\max }=0.984$

Refinement

Refinement on $F^{2}$

Least-squares matrix: full

$R\left[F^{2}>2 \sigma\left(F^{2}\right)\right]=0.055$

$w R\left(F^{2}\right)=0.164$

$S=1.11$

3588 reflections

302 parameters

0 restraints
$D_{\mathrm{x}}=1.471 \mathrm{Mg} \mathrm{m}^{-3}$

Mo $K \alpha$ radiation, $\lambda=0.71073 \AA$

Cell parameters from 9400 reflections

$\theta=2.3-24.0^{\circ}$

$\mu=0.11 \mathrm{~mm}^{-1}$

$T=296 \mathrm{~K}$

Block, brown

$0.35 \times 0.30 \times 0.25 \mathrm{~mm}$

27633 measured reflections

3588 independent reflections 2797 reflections with $I>2 \sigma(I)$

$R_{\text {int }}=0.038$

$\theta_{\text {max }}=25.0^{\circ}, \theta_{\text {min }}=2.3^{\circ}$

$h=-8 \rightarrow 8$

$k=-18 \rightarrow 18$

$l=-20 \rightarrow 20$

Hydrogen site location: mixed

$\mathrm{H}$ atoms treated by a mixture of independent and constrained refinement

$w=1 /\left[\sigma^{2}\left(F_{\mathrm{o}}^{2}\right)+(0.0596 P)^{2}+2.2423 P\right]$

where $P=\left(F_{\mathrm{o}}^{2}+2 F_{\mathrm{c}}{ }^{2}\right) / 3$

$(\Delta / \sigma)_{\max }<0.001$

$\Delta \rho_{\max }=0.29 \mathrm{e} \AA^{-3}$

$\Delta \rho_{\text {min }}=-0.26$ e $\AA^{-3}$ 
Absolute structure: Flack $x$ determined using

1033 quotients $\left[\left(I^{+}\right)-\left(I^{-}\right)\right] /\left[\left(I^{+}\right)+\left(I^{-}\right)\right]$(Parsons et al., 2013)

Absolute structure parameter: 0.5 (4)

\section{Special details}

Geometry. All esds (except the esd in the dihedral angle between two 1.s. planes) are estimated using the full covariance matrix. The cell esds are taken into account individually in the estimation of esds in distances, angles and torsion angles; correlations between esds in cell parameters are only used when they are defined by crystal symmetry. An approximate (isotropic) treatment of cell esds is used for estimating esds involving l.s. planes.

Refinement. The elements in the sample do not have sufficient anomalous scattering power for $\operatorname{Mo}(\mathrm{k} \alpha)$ radiation. Hence the Flack parameter and its standard deviation obtained from refinement have no physical significance.

Fractional atomic coordinates and isotropic or equivalent isotropic displacement parameters $\left(\hat{A}^{2}\right)$

\begin{tabular}{|c|c|c|c|c|}
\hline & $x$ & $y$ & $z$ & $U_{\text {iso }} * / U_{\text {eq }}$ \\
\hline $\mathrm{C} 1$ & $1.0128(8)$ & $0.1849(4)$ & $0.6047(3)$ & $0.0420(13)$ \\
\hline $\mathrm{C} 2$ & $0.9775(8)$ & $0.1140(3)$ & $0.5603(3)$ & $0.0430(13)$ \\
\hline $\mathrm{H} 2$ & 0.9489 & 0.0617 & 0.5830 & $0.052^{*}$ \\
\hline $\mathrm{C} 3$ & $0.9850(7)$ & $0.1217(3)$ & $0.4818(3)$ & $0.0380(12)$ \\
\hline $\mathrm{C} 4$ & $1.0322(7)$ & 0.1979 (3) & $0.4434(3)$ & $0.0360(11)$ \\
\hline $\mathrm{C} 5$ & $1.0660(8)$ & $0.2679(3)$ & $0.4922(3)$ & $0.0443(13)$ \\
\hline H5 & 1.0970 & 0.3204 & 0.4705 & $0.053^{*}$ \\
\hline C6 & $1.0549(8)$ & 0.2615 & $0.5715(3)$ & $0.0495(14)$ \\
\hline H6 & 1.0761 & 0.3094 & 0.6021 & $0.059^{*}$ \\
\hline $\mathrm{C} 7$ & $1.0594(7)$ & $0.2047(3)$ & $0.3605(3)$ & $0.0337(11)$ \\
\hline $\mathrm{C} 8$ & $1.1510(7)$ & $0.1402(3)$ & $0.3218(3)$ & $0.0357(11)$ \\
\hline C9 & $1.1333(8)$ & $0.2196(3)$ & $0.2030(3)$ & $0.0421(13)$ \\
\hline $\mathrm{C} 10$ & $1.0069(7)$ & $0.2807(3)$ & $0.3207(3)$ & $0.0388(12)$ \\
\hline $\mathrm{C} 11$ & $0.9855(12)$ & $0.3574(4)$ & $0.1982(4)$ & $0.072(2)$ \\
\hline H11A & 0.9241 & 0.3963 & 0.2317 & $0.108^{*}$ \\
\hline H11B & 1.0872 & 0.3856 & 0.1766 & $0.108^{*}$ \\
\hline $\mathrm{H} 11 \mathrm{C}$ & 0.9074 & 0.3396 & 0.1576 & $0.108^{*}$ \\
\hline $\mathrm{C} 12$ & $1.2891(9)$ & 0.0820 & $0.2065(3)$ & $0.0569(16)$ \\
\hline H12A & 1.3142 & 0.0369 & 0.2426 & $0.085^{*}$ \\
\hline H12B & 1.2236 & 0.0590 & 0.1639 & $0.085^{*}$ \\
\hline $\mathrm{H} 12 \mathrm{C}$ & 1.3985 & 0.1062 & 0.1884 & $0.085^{*}$ \\
\hline C13 & $1.4554(8)$ & $0.0032(4)$ & $0.5236(3)$ & $0.0486(14)$ \\
\hline H13 & 1.4114 & 0.0499 & 0.5511 & $0.058 *$ \\
\hline $\mathrm{C} 14$ & $1.5694(7)$ & $-0.0536(3)$ & $0.5585(3)$ & $0.0404(12)$ \\
\hline $\mathrm{C} 15$ & $1.6326(7)$ & $-0.1244(4)$ & $0.5152(3)$ & 0.0449 (13) \\
\hline $\mathrm{C} 16$ & $1.5786(8)$ & $-0.1322(4)$ & $0.4383(3)$ & $0.0522(15)$ \\
\hline H16 & 1.6195 & -0.1776 & 0.4084 & $0.063 *$ \\
\hline $\mathrm{C} 17$ & $1.4675(8)$ & $-0.0738(4)$ & $0.4083(3)$ & $0.0546(15)$ \\
\hline H17 & 1.4313 & -0.0788 & 0.3573 & $0.065^{*}$ \\
\hline $\mathrm{C} 18$ & $1.6259(8)$ & $-0.0443(4)$ & $0.6357(3)$ & $0.0554(15)$ \\
\hline H18 & 1.5888 & 0.0026 & 0.6647 & $0.066^{*}$ \\
\hline C19 & $1.7348(9)$ & $-0.1043(6)$ & $0.6669(4)$ & $0.069(2)$ \\
\hline H19 & 1.7689 & -0.0991 & 0.7181 & $0.083^{*}$ \\
\hline
\end{tabular}




\begin{tabular}{lllll}
$\mathrm{C} 20$ & $1.7960(10)$ & $-0.1728(5)$ & $0.6242(4)$ & $0.074(2)$ \\
$\mathrm{H} 20$ & 1.8716 & -0.2126 & 0.6471 & $0.088^{*}$ \\
$\mathrm{C} 21$ & $1.7487(9)$ & $-0.1835(4)$ & $0.5502(4)$ & $0.0642(18)$ \\
$\mathrm{H} 21$ & 1.7926 & -0.2298 & 0.5223 & $0.077^{*}$ \\
$\mathrm{~N} 1$ & $1.0035(8)$ & $0.1760(4)$ & $0.6875(3)$ & $0.0628(15)$ \\
$\mathrm{N} 2$ & $0.9243(7)$ & $0.0456(3)$ & $0.4385(3)$ & $0.0496(12)$ \\
$\mathrm{N} 3$ & $1.0427(7)$ & $0.2826(3)$ & $0.2415(2)$ & $0.0435(11)$ \\
$\mathrm{N} 4$ & $1.1845(6)$ & $0.1485(3)$ & $0.2441(2)$ & $0.0389(10)$ \\
$\mathrm{N} 5$ & $1.4081(7)$ & $-0.0079(3)$ & $0.4514(3)$ & $0.0508(13)$ \\
O1 & $1.0316(9)$ & $0.2406(4)$ & $0.7261(3)$ & $0.0887(18)$ \\
O2 & $0.9721(10)$ & $0.1063(4)$ & $0.7153(3)$ & $0.099(2)$ \\
O3 & $0.8197(6)$ & $0.0553(3)$ & $0.3865(3)$ & $0.0632(12)$ \\
O4 & $0.9781(7)$ & $-0.0246(3)$ & $0.4618(3)$ & $0.0690(14)$ \\
O5 & $0.9298(6)$ & $0.3428(3)$ & $0.3499(2)$ & $0.0578(11)$ \\
O6 & $1.1665(7)$ & $0.2254(3)$ & $0.1336(2)$ & $0.0643(12)$ \\
O7 & $1.2084(6)$ & $0.0724(2)$ & $0.3535(2)$ & $0.0534(11)$ \\
$\mathrm{H} 5 \mathrm{~A}$ & $1.330(8)$ & $0.032(4)$ & $0.431(3)$ & $0.043(15)^{*}$ \\
\hline
\end{tabular}

Atomic displacement parameters $\left(\AA^{2}\right)$

\begin{tabular}{|c|c|c|c|c|c|c|}
\hline & $U^{11}$ & $U^{22}$ & $U^{33}$ & $U^{12}$ & $U^{13}$ & $U^{23}$ \\
\hline $\mathrm{C} 1$ & $0.050(3)$ & $0.052(3)$ & $0.025(2)$ & $0.002(3)$ & $0.002(2)$ & $-0.005(2)$ \\
\hline $\mathrm{C} 2$ & $0.053(3)$ & $0.039(3)$ & $0.037(3)$ & $-0.001(3)$ & -0.001 & $0.005(2)$ \\
\hline $\mathrm{C} 3$ & $0.042(3)$ & $0.037(3)$ & $0.035(3)$ & $-0.002(2)$ & $0.000(2)$ & $-0.002(2)$ \\
\hline $\mathrm{C} 4$ & $0.036(3)$ & 0.039 (3) & $0.034(3)$ & $0.002(2)$ & $-0.005(2)$ & $-0.003(2)$ \\
\hline $\mathrm{C} 5$ & $0.050(3)$ & $0.040(3)$ & $0.044(3)$ & $0.000(3)$ & $-0.004(3)$ & $0.002(2)$ \\
\hline C6 & $0.054(3)$ & $0.051(3)$ & $0.043(3)$ & $0.002(3)$ & -0.010 & -0.018 \\
\hline $\mathrm{C} 7$ & $0.041(3)$ & $0.030(2)$ & $0.030(3)$ & $-0.002(2)$ & $-0.004(2)$ & $0.000(2)$ \\
\hline $\mathrm{C} 8$ & $0.035(3)$ & $0.039(3)$ & $0.033(3)$ & $0.000(2)$ & $-0.004(2)$ & $0.002(2)$ \\
\hline C9 & 0.047 (3) & $0.042(3)$ & $0.038(3)$ & $-0.013(3)$ & $0.001(2)$ & $0.006(2)$ \\
\hline $\mathrm{C} 10$ & $0.042(3)$ & $0.032(3)$ & $0.043(3)$ & $-0.004(2)$ & $-0.001(2)$ & $0.002(2)$ \\
\hline $\mathrm{C} 11$ & $0.108(6)$ & $0.053(4)$ & $0.054(4)$ & $0.004(4)$ & $-0.006(4)$ & $0.015(3)$ \\
\hline $\mathrm{C} 12$ & $0.059(4)$ & $0.065(4)$ & $0.047(3)$ & 0.008 & $0.012(3)$ & $0.000(3)$ \\
\hline C13 & $0.048(3)$ & $0.042(3)$ & $0.055(4)$ & $0.004(3)$ & $0.003(3)$ & $-0.004(3)$ \\
\hline $\mathrm{C} 14$ & $0.037(3)$ & 0.039 (3) & $0.045(3)$ & $-0.002(2)$ & $0.002(2)$ & $0.004(2)$ \\
\hline $\mathrm{C} 15$ & $0.039(3)$ & $0.046(3)$ & 0.049 (3) & $0.005(3)$ & $0.002(3)$ & $0.008(3)$ \\
\hline $\mathrm{C} 16$ & $0.051(3)$ & $0.052(3)$ & $0.054(4)$ & $0.007(3)$ & $0.002(3)$ & $-0.011(3)$ \\
\hline $\mathrm{C} 17$ & $0.055(4)$ & $0.064(4)$ & $0.045(3)$ & $-0.002(3)$ & $-0.006(3)$ & $0.004(3)$ \\
\hline C18 & $0.054(4)$ & $0.063(4)$ & $0.049(4)$ & $-0.006(3)$ & -0.001 & $-0.004(3)$ \\
\hline C19 & $0.053(4)$ & $0.105(6)$ & 0.049 (4) & $-0.014(4)$ & -0.013 & $0.012(4)$ \\
\hline $\mathrm{C} 20$ & $0.066(4)$ & $0.082(5)$ & $0.073(5)$ & $0.019(4)$ & $-0.013(4)$ & $0.021(4)$ \\
\hline $\mathrm{C} 21$ & $0.062(4)$ & 0.057 (4) & $0.074(5)$ & $0.020(3)$ & $-0.007(4)$ & $0.008(3)$ \\
\hline N1 & $0.070(4)$ & $0.081(4)$ & 0.037 (3) & $0.018(3)$ & -0.002 & $-0.004(3)$ \\
\hline N2 & $0.057(3)$ & $0.041(3)$ & $0.051(3)$ & $-0.010(2)$ & $0.009(3)$ & $-0.007(2)$ \\
\hline N3 & $0.062(3)$ & $0.033(2)$ & $0.036(2)$ & $0.002(2)$ & $-0.002(2)$ & 0.0105 (19) \\
\hline N4 & $0.040(2)$ & $0.042(2)$ & $0.035(2)$ & $0.003(2)$ & $0.0034(19)$ & 0.0010 (19) \\
\hline N5 & $0.051(3)$ & $0.048(3)$ & $0.054(3)$ & $0.008(2)$ & $-0.008(2)$ & $0.008(3)$ \\
\hline $\mathrm{O} 1$ & $0.122(5)$ & $0.099(4)$ & $0.045(3)$ & $0.009(4)$ & $-0.011(3)$ & -0.026 \\
\hline
\end{tabular}




\begin{tabular}{|c|c|c|c|c|c|c|}
\hline $\mathrm{O} 2$ & $0.158(6)$ & 0.094 (4) & $0.046(3)$ & $-0.004(4)$ & 0.003 (4) & $0.015(3)$ \\
\hline $\mathrm{O} 3$ & $0.066(3)$ & 0.065 & $0.058(3)$ & $-0.014(2)$ & $-0.012(2)$ & $-0.016(2)$ \\
\hline $\mathrm{O} 4$ & 0.099 (4) & $0.040(2)$ & $0.068(3)$ & -0.005 (2) & $0.012(3)$ & $-0.001(2)$ \\
\hline O5 & $0.076(3)$ & $0.044(2)$ & $0.054(2)$ & $0.010(2)$ & $-0.002(2)$ & $0.006(2)$ \\
\hline O6 & $0.086(3)$ & 0.063 & $0.044(3)$ & $-0.011(3)$ & 0.009 (2) & $0.013(2)$ \\
\hline $\mathrm{O} 7$ & $0.060(3)$ & 0.055 & $0.045(2)$ & 0.017 (2) & $0.003(2)$ & $0.007(2)$ \\
\hline
\end{tabular}

Geometric parameters $\left(\AA,{ }^{o}\right)$

\begin{tabular}{|c|c|c|c|}
\hline $\mathrm{C} 1-\mathrm{C} 6$ & $1.362(8)$ & $\mathrm{C} 12-\mathrm{H} 12 \mathrm{~A}$ & 0.9600 \\
\hline $\mathrm{C} 1-\mathrm{C} 2$ & $1.372(7)$ & $\mathrm{C} 12-\mathrm{H} 12 \mathrm{~B}$ & 0.9600 \\
\hline $\mathrm{C} 1-\mathrm{N} 1$ & $1.448(7)$ & $\mathrm{C} 12-\mathrm{H} 12 \mathrm{C}$ & 0.9600 \\
\hline $\mathrm{C} 2-\mathrm{C} 3$ & $1.372(7)$ & $\mathrm{C} 13-\mathrm{N} 5$ & $1.316(7)$ \\
\hline $\mathrm{C} 2-\mathrm{H} 2$ & 0.9300 & $\mathrm{C} 13-\mathrm{C} 14$ & $1.374(8)$ \\
\hline $\mathrm{C} 3-\mathrm{C} 4$ & $1.406(7)$ & $\mathrm{C} 13-\mathrm{H} 13$ & 0.9300 \\
\hline $\mathrm{C} 3-\mathrm{N} 2$ & $1.476(7)$ & $\mathrm{C} 14-\mathrm{C} 18$ & $1.415(8)$ \\
\hline $\mathrm{C} 4-\mathrm{C} 5$ & $1.404(7)$ & $\mathrm{C} 14-\mathrm{C} 15$ & $1.418(7)$ \\
\hline $\mathrm{C} 4-\mathrm{C} 7$ & $1.460(7)$ & $\mathrm{C} 15-\mathrm{C} 16$ & $1.402(8)$ \\
\hline $\mathrm{C} 5-\mathrm{C} 6$ & $1.385(8)$ & $\mathrm{C} 15-\mathrm{C} 21$ & $1.408(8)$ \\
\hline $\mathrm{C} 5-\mathrm{H} 5$ & 0.9300 & $\mathrm{C} 16-\mathrm{C} 17$ & $1.342(8)$ \\
\hline $\mathrm{C} 6-\mathrm{H} 6$ & 0.9300 & $\mathrm{C} 16-\mathrm{H} 16$ & 0.9300 \\
\hline $\mathrm{C} 7-\mathrm{C} 8$ & $1.391(7)$ & $\mathrm{C} 17-\mathrm{N} 5$ & $1.346(8)$ \\
\hline $\mathrm{C} 7-\mathrm{C} 10$ & $1.425(7)$ & $\mathrm{C} 17-\mathrm{H} 17$ & 0.9300 \\
\hline $\mathrm{C} 8-\mathrm{O} 7$ & $1.267(6)$ & $\mathrm{C} 18-\mathrm{C} 19$ & $1.357(10)$ \\
\hline $\mathrm{C} 8-\mathrm{N} 4$ & $1.381(6)$ & $\mathrm{C} 18-\mathrm{H} 18$ & 0.9300 \\
\hline $\mathrm{C} 9-\mathrm{O} 6$ & $1.235(6)$ & $\mathrm{C} 19-\mathrm{C} 20$ & $1.379(10)$ \\
\hline $\mathrm{C} 9-\mathrm{N} 3$ & $1.369(7)$ & C19-H19 & 0.9300 \\
\hline $\mathrm{C} 9-\mathrm{N} 4$ & $1.373(7)$ & $\mathrm{C} 20-\mathrm{C} 21$ & $1.346(10)$ \\
\hline $\mathrm{C} 10-\mathrm{O} 5$ & $1.236(6)$ & $\mathrm{C} 20-\mathrm{H} 20$ & 0.9300 \\
\hline $\mathrm{C} 10-\mathrm{N} 3$ & $1.404(7)$ & $\mathrm{C} 21-\mathrm{H} 21$ & 0.9300 \\
\hline $\mathrm{C} 11-\mathrm{N} 3$ & $1.452(7)$ & $\mathrm{N} 1-\mathrm{O} 2$ & $1.211(7)$ \\
\hline C11-H11A & 0.9600 & $\mathrm{~N} 1-\mathrm{O} 1$ & $1.228(8)$ \\
\hline C11-H11B & 0.9600 & $\mathrm{~N} 2-\mathrm{O} 3$ & $1.209(6)$ \\
\hline $\mathrm{C} 11-\mathrm{H} 11 \mathrm{C}$ & 0.9600 & $\mathrm{~N} 2-\mathrm{O} 4$ & $1.234(6)$ \\
\hline $\mathrm{C} 12-\mathrm{N} 4$ & $1.455(7)$ & $\mathrm{N} 5-\mathrm{H} 5 \mathrm{~A}$ & $0.93(6)$ \\
\hline $\mathrm{C} 6-\mathrm{C} 1-\mathrm{C} 2$ & $120.6(5)$ & $\mathrm{N} 5-\mathrm{C} 13-\mathrm{C} 14$ & $120.4(5)$ \\
\hline $\mathrm{C} 6-\mathrm{C} 1-\mathrm{N} 1$ & $121.1(5)$ & $\mathrm{N} 5-\mathrm{C} 13-\mathrm{H} 13$ & 119.8 \\
\hline $\mathrm{C} 2-\mathrm{C} 1-\mathrm{N} 1$ & $118.3(5)$ & $\mathrm{C} 14-\mathrm{C} 13-\mathrm{H} 13$ & 119.8 \\
\hline $\mathrm{C} 3-\mathrm{C} 2-\mathrm{C} 1$ & $118.9(5)$ & $\mathrm{C} 13-\mathrm{C} 14-\mathrm{C} 18$ & $122.8(6)$ \\
\hline $\mathrm{C} 3-\mathrm{C} 2-\mathrm{H} 2$ & 120.6 & $\mathrm{C} 13-\mathrm{C} 14-\mathrm{C} 15$ & $118.3(5)$ \\
\hline $\mathrm{C} 1-\mathrm{C} 2-\mathrm{H} 2$ & 120.6 & $\mathrm{C} 18-\mathrm{C} 14-\mathrm{C} 15$ & $118.9(5)$ \\
\hline $\mathrm{C} 2-\mathrm{C} 3-\mathrm{C} 4$ & $123.8(5)$ & $\mathrm{C} 16-\mathrm{C} 15-\mathrm{C} 21$ & $122.4(6)$ \\
\hline $\mathrm{C} 2-\mathrm{C} 3-\mathrm{N} 2$ & $115.1(5)$ & $\mathrm{C} 16-\mathrm{C} 15-\mathrm{C} 14$ & $118.5(5)$ \\
\hline $\mathrm{C} 4-\mathrm{C} 3-\mathrm{N} 2$ & $120.9(5)$ & $\mathrm{C} 21-\mathrm{C} 15-\mathrm{C} 14$ & $119.1(6)$ \\
\hline $\mathrm{C} 5-\mathrm{C} 4-\mathrm{C} 3$ & $114.5(5)$ & $\mathrm{C} 17-\mathrm{C} 16-\mathrm{C} 15$ & $119.5(6)$ \\
\hline $\mathrm{C} 5-\mathrm{C} 4-\mathrm{C} 7$ & $121.0(5)$ & $\mathrm{C} 17-\mathrm{C} 16-\mathrm{H} 16$ & 120.2 \\
\hline $\mathrm{C} 3-\mathrm{C} 4-\mathrm{C} 7$ & $124.4(4)$ & $\mathrm{C} 15-\mathrm{C} 16-\mathrm{H} 16$ & 120.2 \\
\hline
\end{tabular}




\begin{tabular}{|c|c|c|c|}
\hline $\mathrm{C} 6-\mathrm{C} 5-\mathrm{C} 4$ & $122.3(5)$ & $\mathrm{C} 16-\mathrm{C} 17-\mathrm{N} 5$ & $120.5(6)$ \\
\hline $\mathrm{C} 6-\mathrm{C} 5-\mathrm{H} 5$ & 118.9 & $\mathrm{C} 16-\mathrm{C} 17-\mathrm{H} 17$ & 119.8 \\
\hline $\mathrm{C} 4-\mathrm{C} 5-\mathrm{H} 5$ & 118.9 & $\mathrm{~N} 5-\mathrm{C} 17-\mathrm{H} 17$ & 119.8 \\
\hline $\mathrm{C} 1-\mathrm{C} 6-\mathrm{C} 5$ & $120.0(5)$ & $\mathrm{C} 19-\mathrm{C} 18-\mathrm{C} 14$ & $119.5(6)$ \\
\hline $\mathrm{C} 1-\mathrm{C} 6-\mathrm{H} 6$ & 120.0 & $\mathrm{C} 19-\mathrm{C} 18-\mathrm{H} 18$ & 120.3 \\
\hline $\mathrm{C} 5-\mathrm{C} 6-\mathrm{H} 6$ & 120.0 & $\mathrm{C} 14-\mathrm{C} 18-\mathrm{H} 18$ & 120.3 \\
\hline $\mathrm{C} 8-\mathrm{C} 7-\mathrm{C} 10$ & $120.1(4)$ & $\mathrm{C} 18-\mathrm{C} 19-\mathrm{C} 20$ & $121.2(6)$ \\
\hline $\mathrm{C} 8-\mathrm{C} 7-\mathrm{C} 4$ & $119.6(4)$ & $\mathrm{C} 18-\mathrm{C} 19-\mathrm{H} 19$ & 119.4 \\
\hline $\mathrm{C} 10-\mathrm{C} 7-\mathrm{C} 4$ & $120.0(4)$ & $\mathrm{C} 20-\mathrm{C} 19-\mathrm{H} 19$ & 119.4 \\
\hline $\mathrm{O} 7-\mathrm{C} 8-\mathrm{N} 4$ & $116.1(4)$ & $\mathrm{C} 21-\mathrm{C} 20-\mathrm{C} 19$ & $121.5(7)$ \\
\hline $\mathrm{O} 7-\mathrm{C} 8-\mathrm{C} 7$ & $124.1(4)$ & $\mathrm{C} 21-\mathrm{C} 20-\mathrm{H} 20$ & 119.3 \\
\hline $\mathrm{N} 4-\mathrm{C} 8-\mathrm{C} 7$ & $119.8(4)$ & $\mathrm{C} 19-\mathrm{C} 20-\mathrm{H} 20$ & 119.3 \\
\hline $\mathrm{O} 6-\mathrm{C} 9-\mathrm{N} 3$ & $121.8(5)$ & $\mathrm{C} 20-\mathrm{C} 21-\mathrm{C} 15$ & $119.9(7)$ \\
\hline $\mathrm{O} 6-\mathrm{C} 9-\mathrm{N} 4$ & $120.8(5)$ & $\mathrm{C} 20-\mathrm{C} 21-\mathrm{H} 21$ & 120.1 \\
\hline $\mathrm{N} 3-\mathrm{C} 9-\mathrm{N} 4$ & $117.5(4)$ & $\mathrm{C} 15-\mathrm{C} 21-\mathrm{H} 21$ & 120.1 \\
\hline $\mathrm{O} 5-\mathrm{C} 10-\mathrm{N} 3$ & $118.4(5)$ & $\mathrm{O} 2-\mathrm{N} 1-\mathrm{O} 1$ & $123.3(6)$ \\
\hline $\mathrm{O} 5-\mathrm{C} 10-\mathrm{C} 7$ & $125.4(5)$ & $\mathrm{O} 2-\mathrm{N} 1-\mathrm{C} 1$ & $119.5(6)$ \\
\hline $\mathrm{N} 3-\mathrm{C} 10-\mathrm{C} 7$ & $116.1(5)$ & $\mathrm{O} 1-\mathrm{N} 1-\mathrm{C} 1$ & $117.2(6)$ \\
\hline $\mathrm{N} 3-\mathrm{C} 11-\mathrm{H} 11 \mathrm{~A}$ & 109.5 & $\mathrm{O} 3-\mathrm{N} 2-\mathrm{O} 4$ & $124.8(5)$ \\
\hline N3-C11-H11B & 109.5 & $\mathrm{O} 3-\mathrm{N} 2-\mathrm{C} 3$ & $118.9(5)$ \\
\hline $\mathrm{H} 11 \mathrm{~A}-\mathrm{C} 11-\mathrm{H} 11 \mathrm{~B}$ & 109.5 & $\mathrm{O} 4-\mathrm{N} 2-\mathrm{C} 3$ & $116.2(5)$ \\
\hline $\mathrm{N} 3-\mathrm{C} 11-\mathrm{H} 11 \mathrm{C}$ & 109.5 & $\mathrm{C} 9-\mathrm{N} 3-\mathrm{C} 10$ & $124.1(4)$ \\
\hline $\mathrm{H} 11 \mathrm{~A}-\mathrm{C} 11-\mathrm{H} 11 \mathrm{C}$ & 109.5 & $\mathrm{C} 9-\mathrm{N} 3-\mathrm{C} 11$ & $117.9(5)$ \\
\hline $\mathrm{H} 11 \mathrm{~B}-\mathrm{C} 11-\mathrm{H} 11 \mathrm{C}$ & 109.5 & $\mathrm{C} 10-\mathrm{N} 3-\mathrm{C} 11$ & $118.0(5)$ \\
\hline N4-C12-H12A & 109.5 & $\mathrm{C} 9-\mathrm{N} 4-\mathrm{C} 8$ & $122.3(4)$ \\
\hline $\mathrm{N} 4-\mathrm{C} 12-\mathrm{H} 12 \mathrm{~B}$ & 109.5 & $\mathrm{C} 9-\mathrm{N} 4-\mathrm{C} 12$ & $119.4(4)$ \\
\hline $\mathrm{H} 12 \mathrm{~A}-\mathrm{C} 12-\mathrm{H} 12 \mathrm{~B}$ & 109.5 & $\mathrm{C} 8-\mathrm{N} 4-\mathrm{C} 12$ & $118.2(4)$ \\
\hline $\mathrm{N} 4-\mathrm{C} 12-\mathrm{H} 12 \mathrm{C}$ & 109.5 & $\mathrm{C} 13-\mathrm{N} 5-\mathrm{C} 17$ & $122.8(5)$ \\
\hline $\mathrm{H} 12 \mathrm{~A}-\mathrm{C} 12-\mathrm{H} 12 \mathrm{C}$ & 109.5 & $\mathrm{C} 13-\mathrm{N} 5-\mathrm{H} 5 \mathrm{~A}$ & $117(3)$ \\
\hline $\mathrm{H} 12 \mathrm{~B}-\mathrm{C} 12-\mathrm{H} 12 \mathrm{C}$ & 109.5 & $\mathrm{C} 17-\mathrm{N} 5-\mathrm{H} 5 \mathrm{~A}$ & $121(3)$ \\
\hline $\mathrm{C} 6-\mathrm{C} 1-\mathrm{C} 2-\mathrm{C} 3$ & $-0.1(9)$ & $\mathrm{C} 13-\mathrm{C} 14-\mathrm{C} 18-\mathrm{C} 19$ & $178.2(6)$ \\
\hline $\mathrm{N} 1-\mathrm{C} 1-\mathrm{C} 2-\mathrm{C} 3$ & $179.7(5)$ & $\mathrm{C} 15-\mathrm{C} 14-\mathrm{C} 18-\mathrm{C} 19$ & $-1.8(9)$ \\
\hline $\mathrm{C} 1-\mathrm{C} 2-\mathrm{C} 3-\mathrm{C} 4$ & $1.9(9)$ & $\mathrm{C} 14-\mathrm{C} 18-\mathrm{C} 19-\mathrm{C} 20$ & $2.1(10)$ \\
\hline $\mathrm{C} 1-\mathrm{C} 2-\mathrm{C} 3-\mathrm{N} 2$ & $-172.9(5)$ & $\mathrm{C} 18-\mathrm{C} 19-\mathrm{C} 20-\mathrm{C} 21$ & $-0.8(12)$ \\
\hline $\mathrm{C} 2-\mathrm{C} 3-\mathrm{C} 4-\mathrm{C} 5$ & $-2.1(8)$ & $\mathrm{C} 19-\mathrm{C} 20-\mathrm{C} 21-\mathrm{C} 15$ & $-0.8(11)$ \\
\hline $\mathrm{N} 2-\mathrm{C} 3-\mathrm{C} 4-\mathrm{C} 5$ & $172.5(5)$ & $\mathrm{C} 16-\mathrm{C} 15-\mathrm{C} 21-\mathrm{C} 20$ & $-180.0(7)$ \\
\hline $\mathrm{C} 2-\mathrm{C} 3-\mathrm{C} 4-\mathrm{C} 7$ & $173.1(5)$ & $\mathrm{C} 14-\mathrm{C} 15-\mathrm{C} 21-\mathrm{C} 20$ & $0.9(9)$ \\
\hline $\mathrm{N} 2-\mathrm{C} 3-\mathrm{C} 4-\mathrm{C} 7$ & $-12.4(8)$ & $\mathrm{C} 6-\mathrm{C} 1-\mathrm{N} 1-\mathrm{O} 2$ & $-177.3(7)$ \\
\hline $\mathrm{C} 3-\mathrm{C} 4-\mathrm{C} 5-\mathrm{C} 6$ & $0.5(8)$ & $\mathrm{C} 2-\mathrm{C} 1-\mathrm{N} 1-\mathrm{O} 2$ & $2.9(10)$ \\
\hline $\mathrm{C} 7-\mathrm{C} 4-\mathrm{C} 5-\mathrm{C} 6$ & $-174.8(6)$ & $\mathrm{C} 6-\mathrm{C} 1-\mathrm{N} 1-\mathrm{O} 1$ & $1.3(9)$ \\
\hline $\mathrm{C} 2-\mathrm{C} 1-\mathrm{C} 6-\mathrm{C} 5$ & $-1.4(9)$ & $\mathrm{C} 2-\mathrm{C} 1-\mathrm{N} 1-\mathrm{O} 1$ & $-178.4(6)$ \\
\hline $\mathrm{N} 1-\mathrm{C} 1-\mathrm{C} 6-\mathrm{C} 5$ & $178.9(5)$ & $\mathrm{C} 2-\mathrm{C} 3-\mathrm{N} 2-\mathrm{O} 3$ & $131.3(5)$ \\
\hline $\mathrm{C} 4-\mathrm{C} 5-\mathrm{C} 6-\mathrm{C} 1$ & $1.2(9)$ & $\mathrm{C} 4-\mathrm{C} 3-\mathrm{N} 2-\mathrm{O} 3$ & $-43.7(8)$ \\
\hline $\mathrm{C} 5-\mathrm{C} 4-\mathrm{C} 7-\mathrm{C} 8$ & $132.9(5)$ & $\mathrm{C} 2-\mathrm{C} 3-\mathrm{N} 2-\mathrm{O} 4$ & $-44.6(7)$ \\
\hline $\mathrm{C} 3-\mathrm{C} 4-\mathrm{C} 7-\mathrm{C} 8$ & $-41.9(8)$ & $\mathrm{C} 4-\mathrm{C} 3-\mathrm{N} 2-\mathrm{O} 4$ & $140.4(5)$ \\
\hline $\mathrm{C} 5-\mathrm{C} 4-\mathrm{C} 7-\mathrm{C} 10$ & $-41.3(7)$ & $\mathrm{O} 6-\mathrm{C} 9-\mathrm{N} 3-\mathrm{C} 10$ & $-177.7(5)$ \\
\hline $\mathrm{C} 3-\mathrm{C} 4-\mathrm{C} 7-\mathrm{C} 10$ & $143.8(5)$ & $\mathrm{N} 4-\mathrm{C} 9-\mathrm{N} 3-\mathrm{C} 10$ & $3.2(8)$ \\
\hline
\end{tabular}




$\begin{array}{llll}\mathrm{C} 10-\mathrm{C} 7-\mathrm{C} 8-\mathrm{O} 7 & 177.1(5) & \mathrm{O} 6-\mathrm{C} 9-\mathrm{N} 3-\mathrm{C} 11 & 0.2(9) \\ \mathrm{C} 4-\mathrm{C} 7-\mathrm{C} 8-\mathrm{O} 7 & 2.9(8) & \mathrm{N} 4-\mathrm{C} 9-\mathrm{N} 3-\mathrm{C} 11 & -179.0(6) \\ \mathrm{C} 10-\mathrm{C} 7-\mathrm{C} 8-\mathrm{N} 4 & -2.6(7) & \mathrm{O} 5-\mathrm{C} 10-\mathrm{N} 3-\mathrm{C} 9 & -4.4(8) \\ \mathrm{C} 4-\mathrm{C} 7-\mathrm{C} 8-\mathrm{N} 4 & -176.8(5) & \mathrm{C} 7-\mathrm{C} 10-\mathrm{N} 3-\mathrm{C} 9 & -0.5(8) \\ \mathrm{C} 8-\mathrm{C} 7-\mathrm{C} 10-\mathrm{O} 5 & -177.8(5) & \mathrm{O} 5-\mathrm{C} 10-\mathrm{N} 3-\mathrm{C} 11 & 177.7(5) \\ \mathrm{C} 4-\mathrm{C} 7-\mathrm{C} 10-\mathrm{O} 5 & -3.6(8) & \mathrm{O} 7-\mathrm{C} 10-\mathrm{C} 9-\mathrm{N} 4-\mathrm{C} 11 & 179.4(5) \\ \mathrm{C} 8-\mathrm{C} 7-\mathrm{C} 10-\mathrm{N} 3 & 4.0(7) & \mathrm{N} 3-\mathrm{C} 9-\mathrm{N} 4-\mathrm{C} 8 & -1.4(8) \\ \mathrm{C} 4-\mathrm{C} 7-\mathrm{C} 10-\mathrm{N} 3 & 178.2(5) & \mathrm{N} 3-\mathrm{C} 9-\mathrm{N} 4-\mathrm{C} 12 & -176(8) \\ \mathrm{N} 5-\mathrm{C} 13-\mathrm{C} 14-\mathrm{C} 18 & 179.4(6) & \mathrm{O} 7-\mathrm{C} 8-\mathrm{N} 4-\mathrm{C} 12 & -178.5(5) \\ \mathrm{N} 5-\mathrm{C} 13-\mathrm{C} 14-\mathrm{C} 15 & -0.6(8) & \mathrm{C} 7-\mathrm{C} 8-\mathrm{N} 4-\mathrm{C} 9 & 1.2(7) \\ \mathrm{C} 13-\mathrm{C} 14-\mathrm{C} 15-\mathrm{C} 16 & 1.2(8) & \mathrm{O} 7-\mathrm{C} 8-\mathrm{N} 4-\mathrm{C} 12 & -3.1(7) \\ \mathrm{C} 18-\mathrm{C} 14-\mathrm{C} 15-\mathrm{C} 16 & -178.8(5) & \mathrm{C} 14-\mathrm{C} 8-\mathrm{N} 4-\mathrm{C} 12 & 176.7(5) \\ \mathrm{C} 13-\mathrm{C} 14-\mathrm{C} 15-\mathrm{C} 21 & -179.7(5) & \mathrm{C} 16-\mathrm{C} 17-\mathrm{N} 5-\mathrm{C}-\mathrm{C} 13 & -0.3(9) \\ \mathrm{C} 18-\mathrm{C} 14-\mathrm{C} 15-\mathrm{C} 21 & 0.4(8) & & 0.6(9) \\ \mathrm{C} 21-\mathrm{C} 15-\mathrm{C} 16-\mathrm{C} 17 & 179.9(6) & & \end{array}$

Hydrogen-bond geometry $\left(\AA,{ }^{\circ}\right)$

\begin{tabular}{lllll}
\hline$D-\mathrm{H} \cdots A$ & $D-\mathrm{H}$ & $\mathrm{H} \cdots A$ & $D \cdots A$ & $D-\mathrm{H} \cdots A$ \\
\hline $\mathrm{N} 5-\mathrm{H} 5 A \cdots \mathrm{O} 7$ & $0.93(6)$ & $1.74(6)$ & $2.592(6)$ & $150(5)$ \\
$\mathrm{C} 13-\mathrm{H} 13 \cdots \mathrm{O} 5^{\mathrm{i}}$ & 0.93 & 2.40 & $3.260(7)$ & 153 \\
$\mathrm{C} 16-\mathrm{H} 16 \cdots \mathrm{O}^{\mathrm{ii}}$ & 0.93 & 2.33 & $3.187(7)$ & 154 \\
$\mathrm{C} 17-\mathrm{H} 17 \cdots \mathrm{O} 2^{\mathrm{iii}}$ & 0.93 & 2.61 & $3.424(8)$ & 146 \\
\hline
\end{tabular}

Symmetry codes: (i) $x+1 / 2,-y+1 / 2,-z+1$; (ii) $-x+3, y-1 / 2,-z+1 / 2$; (iii) $-x+5 / 2,-y, z-1 / 2$. 